\title{
Application of Hypoxic Exposure Combined with Osteogenic Induction for the Enhancement of Multiple Osteoinductive Capabilities in Rat Mesenchymal Cells
}

\author{
Naoaki Saito1,2,3, Hiroko Kato ${ }^{4}$, Yosuke Akiba ${ }^{5}$, Yuko Hara' ${ }^{2}$, Taku Kojima ${ }^{1}$, Michiko Yoshizawa ${ }^{1}$, \\ Atsushi Ohazama ${ }^{3}$, Takeyasu Maeda ${ }^{4}$, Tadaharu Kobayashi' ${ }^{1}$ Kenji Izumi2 ${ }^{*}$ \\ ${ }^{1}$ Division of Reconstructive Surgery for Oral and Maxillofacial Region, Department of Tissue Regeneration and Reconstruction, \\ Niigata University Graduate School of Medical and Dental Sciences, Niigata, Japan \\ ${ }^{2}$ Division of Biomimetics, Department of Oral Health Science, Niigata University Graduate School of Medical and Dental Sciences, \\ Niigata, Japan \\ ${ }^{3}$ Division of Oral Anatomy, Department of Oral Biological Science, Niigata University Graduate School of Medical and Dental \\ Sciences, Niigata, Japan \\ ${ }^{4}$ Research Center for Advanced Oral Science, Niigata University Graduate School of Medical and Dental Sciences, Niigata, Japan \\ ${ }^{5}$ Division of Bio-Prosthodontics, Department of Oral Health Science, Niigata University Graduate School of Medical and Dental \\ Sciences, Niigata, Japan \\ Email: *izumik@dent.niigata-u.ac.jp
}

How to cite this paper: Saito, N., Kato, H., Akiba, Y., Hara, Y., Kojima, T., Yoshizawa, M., Ohazama, A., Maeda, T., Kobayashi, T. and Izumi, K. (2018) Application of Hypoxic Exposure Combined with Osteogenic Induction for the Enhancement of Multiple Osteoinductive Capabilities in Rat Mesenchymal Cells. Open Journal of Stomatology, 8, 53-69.

https://doi.org/10.4236/ojst.2018.82005

Received: January 2, 2018

Accepted: February 23, 2018

Published: February 26, 2018

Copyright $\odot 2018$ by authors and Scientific Research Publishing Inc. This work is licensed under the Creative Commons Attribution International License (CC BY 4.0).

http://creativecommons.org/licenses/by/4.0/

(c) (i) Open Access

\begin{abstract}
Objective: The enhancement of multiple functions, including osteogenesis, angiogenesis, and cell recruitment, is required for efficient bone regeneration therapy. Recently, special attention has been focused on the microenvironment of stem cells to facilitate bone regeneration. Herein, we examined the effects of various combinations of hypoxic conditions and osteogenic induction on rat mesenchymal cells, to develop a specific protocol for enhancing the multiple cellular functions beneficial to bone regeneration. Methods: Rat mesenchymal cells, isolated from bone marrow, adipose tissue, and periodontal ligament, were examined. The cells were cultured under varied conditions of $\mathrm{O}_{2}$ tension (hypoxia) and duration and timing of hypoxic exposure, with or without osteogenic induction. Consequently, four different protocols were examined by measuring the gene expression levels of Runx2, Vegfa, and Cxcl12, indicating a capability for osteogenesis, angiogenesis, and cell recruitment, respectively. Finally, the mineralization ability of the rat mesenchymal cells was assessed by quantitating their calcified nodule formation. Results: The simultaneous application of hypoxic exposure and osteogenic induction promoted Vegfa expression in all types of cells, but suppressed Runx2. In contrast, hy-
\end{abstract}


poxic preconditioning, followed by osteogenic induction, did not increase the expression of these genes; in fact, Vegfa expression decreased significantly. Among the various protocols, $0.5 \% \mathrm{O}_{2}$ exposure for $12 \mathrm{~h}$ after osteogenic induction exhibited the largest fold changes of gene expression level, especially of Vegfa. Hypoxic post-conditioning enhanced the formation of calcified nodules in periodontal ligament-derived cells. Conclusion: Short-term hypoxic exposure after osteogenic induction could be used to improve the efficiency of mesenchymal cells for bone regeneration.

\section{Keywords}

Osteogenesis, Hypoxia, Angiogenesis, Cell Recruitment, Mesenchymal Stem Cells

\section{Introduction}

Mastication, speech, and facial aesthetics are often severely compromised by jaw bone defects caused by trauma, inflammatory disease, and benign or malignant tumors in the oral and maxillofacial region. Therefore, recovery of the structural and functional features of the jaw is an extremely important task of maxillofacial surgeons [1] [2]. Currently, although autogenous bone grafting is a standard treatment for jaw bone reconstruction, it may cause donor site morbidity, post-operative complications, healing failure, and insufficient bone volume [2] [3] [4]. For overcoming these problems, tissue engineering technology has a lot of potential, providing an alternative treatment option for autogenous bone grafting in jaw bone reconstruction and bone regeneration therapy [5] [6] [7].

The original principle of bone regeneration therapy was that the transplanted cells could facilitate osteogenesis in host tissues by themselves [8]. However, it has been reconsidered that manipulation of the host tissue environment by a multifaceted approach, such as induction of osteogenic cells, promotion of angiogenesis, and supplementation of growth factors, is also important to promote the survival of transplanted cells and reduce the period of new-bone formation [9]. As a strategy to provide those functions to mesenchymal stem cells (MSCs), stem cell preconditioning in vitro prior to cell transplantation has been studied [10] [11]. For example, heat-shock preconditioning increases the cell survival rate, and tumor necrosis factor-alpha preconditioning enhances the osteogenic differentiation ability of adipose-derived stem cells [12] [13]. Furthermore, hypoxic preconditioning of bone marrow-derived MSCs promotes angiogenesis or neurogenesis in damaged heart and brain tissues [14] [15]. Although hypoxic preconditioning is considered to be beneficial to MSCs to enhance their angiogenic activity and cell induction ability, it is still controversial for bone regeneration therapy because some reports have demonstrated that osteogenic differentiation was accelerated under hypoxia, whereas many others stated that the process was suppressed [16]-[22]. 
As a MSC source, the osteogenic potential is considered to be the most dominant in bone marrow-derived cells (BMDCs) [23] [24]. In comparison, adipose tissue-derived cells (ATDCs) are known to possess weak osteogenic ability, but their advantage is that a relatively large amount of tissue can be harvested with minimal invasiveness [25]. On the other hand, periodontal ligament-derived cells (PDLDCs) have an ability to form new bone comparable to that of BMDCs [26]. In the craniofacial and maxillofacial regions, there are many tissue sources of MSCs other than bone marrow [27]. Thus, there is a need to characterize cellular responses under hypoxic conditions according to the mesenchymal cells derived from different tissue sources. In this study, we examined the effects of various combinational uses of hypoxic conditions and osteogenic induction on rat mesenchymal cells in order to develop a specific protocol for enhancing the multiple cellular functions beneficial to bone regeneration.

\section{Materials and Methods}

\subsection{Rat Bone Marrow-Derived Cells: Isolation and Primary Culture}

Four-week-old male Fisher rats (Charles River Laboratories Japan, Yokohama, Japan) were sacrificed by cervical dislocation under deep anesthesia induced through the intraperitoneal administration of $4 \%$ chloral hydrate. The femur was aseptically removed and the adherent soft tissue was thoroughly debrided. After the epiphyses were removed, a 21-gauge needle attached to a $10-\mathrm{mL}$ syringe was used to bore a small opening through the growth plate on the distal end of the femur. A 10 -mL syringe fitted with a fresh 21-gauge needle was filled with alpha-minimal essential medium ( $\alpha$-MEM; Thermo Fisher Scientific, Waltham, MA, USA) supplemented with 10\% fetal bovine serum (FBS; Thermo Fisher Scientific) and 1\% antibiotics-antimycotics (Thermo Fisher Scientific). The needle was inserted into the previously prepared opening, and a small volume of medium was ejected to expel the bone marrow from the medullary canal. The cells were seeded into T-150 cell culture flasks (Corning Inc., Corning, NY, USA). Culture vessels were placed in a $37^{\circ} \mathrm{C}$ humidified incubator under ambient oxygen with $5 \% \mathrm{CO}_{2}$. The first medium exchange was conducted $24 \mathrm{~h}$ later, for the removal of blood cell components. Adherent cells were used as BMDCs.

\subsection{Rat Adipose Tissue-Derived Cells: Isolation and Primary Culture}

Subcutaneous adipose tissue was collected from the back of 4 -week-old male Fisher rats and then minced with scissors and treated with $1 \%$ collagenase (Wako Pure Chemical Industries, Osaka, Japan) solution for $1 \mathrm{~h}$ at $37^{\circ} \mathrm{C}$. Following the incubation, the collagenase was neutralized with an equal volume of $\alpha$-MEM supplemented with $10 \%$ FBS and $1 \%$ antibiotics-antimycotics. The samples were filtered through a $100-\mu \mathrm{m}$ cell strainer (Corning) and centrifuged at $1300 \mathrm{rpm}$ for $5 \mathrm{~min}$. The cells were then seeded into T-75 cell culture flasks (Corning). 


\subsection{Rat Periodontal Ligament-Derived Cells: Isolation and Primary Culture}

Upper and lower molars were extracted from 4-week-old male Fisher rats and the gum tissue was removed from each tooth under a magnifying glass. The molars were then placed on 100-mm cell culture dishes (Corning), to which $8 \mathrm{~mL}$ of $\alpha$-MEM supplemented with $10 \%$ FBS and $1 \%$ antibiotics-antimycotics was added. Once the cell outgrowth $80 \%$ confluent, the cells were subcultured in T-25 cell culture flasks.

These BMDCs, ATDCs from passages 2 to 3, and PDLDCs from passages 4 to 6 , at an estimated confluency of $80 \%$, were used in the study.

\subsection{Flow Cytometry}

The MSC profiles of the BMDCs, ATDCs, and PDLDCs were determined through study of their expression of specific cell-surface markers by flow cytometry. After $3.0 \times 10^{3}$ of cells had been rinsed with $1 \%$ bovine serum albumin in phosphate-buffered saline, they were incubated with the primary antibody at room temperature for $30 \mathrm{~min}$. The following fluorescein isothiocyanate (FITC)-conjugated antibodies against rat were used: anti-mouse/rat CD29 FITC (1:100), anti-mouse/ rat CD90.1 FITC (1:100), and anti-rat CD45 FITC (1:200) (eBioscience, San Diego, CA, USA). Flow cytometry analysis was performed using a FACS Aria II (BD Bioscience, San Jose, CA, USA).

\subsection{Hypoxic Exposure}

The various tissue-derived cells were cultured in air-tight modular incubator chambers (Billups-Rothenberg, Del Mar, CA, USA). Humidity was supplied by placing an open tissue culture dish containing $10 \mathrm{~mL}$ of sterile distilled water at the bottom of each chamber. Gas mixtures, consisting of $0.5 \%, 2 \%$, or $5 \%$ oxygen, were flushed into the chambers for 2 min to purge the ambient air. The chambers were then placed into an incubator at $37^{\circ} \mathrm{C}$. Cells were fed with the $\alpha$-MEM $\left(0.2 \mathrm{ml} / \mathrm{cm}^{2}\right)$ every other day. Prior to feeding under hypoxic conditions, the fresh complete medium was equilibrated under three different $\mathrm{O}_{2}$ tensions for at least $48 \mathrm{~h}$. For normoxic cell culture, culture vessels were placed in a $37^{\circ} \mathrm{C}$ humidified incubator under ambient oxygen with $5 \% \mathrm{CO}_{2}$.

\subsection{Induction of Osteogenic Differentiation}

For osteogenic differentiation, the cells were cultured in a standard osteogenic induction medium supplemented with $100 \mathrm{nM}$ dexamethasone (Sigma-Aldrich, St. Louis, MO, USA), $10 \mathrm{mM}$ beta-glycerophosphate (Wako Pure Chemical Industries), and $50 \mathrm{mM}$ ascorbic acid (Sigma-Aldrich).

\subsection{Protocols of Hypoxic Exposure and Osteogenic Induction}

To explore and narrow down the most effective conditions for the combinational use of hypoxic exposure and osteogenic induction, we examined four proto- 
cols (detailed in Sections 2.7.1 through 2.7.4) in which different $\mathrm{O}_{2}$ tensions and duration and timing of hypoxic exposure, with or without osteogenic induction, were applied.

The four protocols were examined in order of 1 through 4 every time by analyzing the rat gene expression levels of runt-related transcription factor 2 (Runx2; an essential transcription factor for osteoblast differentiation, and an osteogenic differentiation marker), vascular endothelial growth factor A (Vegfa; an angiogenesis marker), and C-X-C motif chemokine 12 (Cxcl12; a marker of cell recruitment), using the reverse transcription real-time polymerase chain reaction (RT-qPCR).

\subsubsection{Protocol 1: Effects of Simultaneous Treatment of Osteogenic Induction and $2 \% \mathrm{O}_{2}$ Exposure}

Cells were cultured for $96 \mathrm{~h}$ under normoxic $\left(21 \% \mathrm{O}_{2}\right)$ (Figure $1(\mathrm{~A})$-a) or hypoxic $\left(2 \% \mathrm{O}_{2}\right)$ (Figure $1(\mathrm{~A})$-b) conditions without osteogenic induction, or under hypoxia with simultaneous treatment of osteogenic induction (Figure $1(\mathrm{~A})-\mathrm{c})$.

(A)

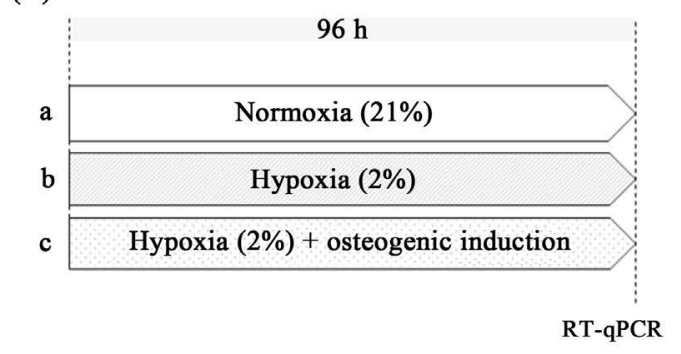

(C)

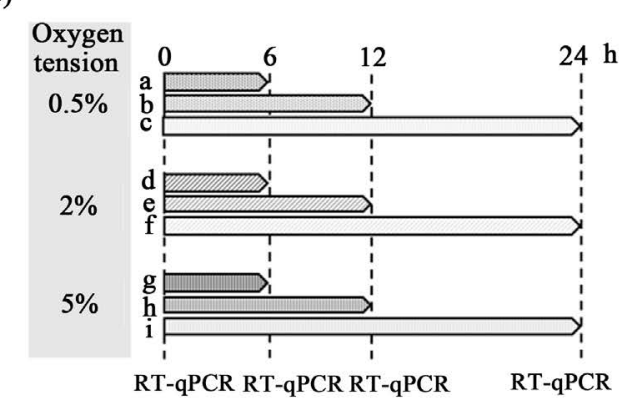

(B)

\begin{tabular}{|c|c|c|}
\hline & $72 \mathrm{~h}$ & $96 \mathrm{~h}$ \\
\hline $\mathbf{a}$ & \multicolumn{2}{|c|}{ Normoxia (21\%) } \\
\hline b & Normoxia (21\%) & Normoxia $(21 \%)+$ osteogenic induction \\
\hline c & Hypoxia (2\%) & Normoxia $(21 \%)+$ osteogenic induction \\
\hline
\end{tabular}

(D)

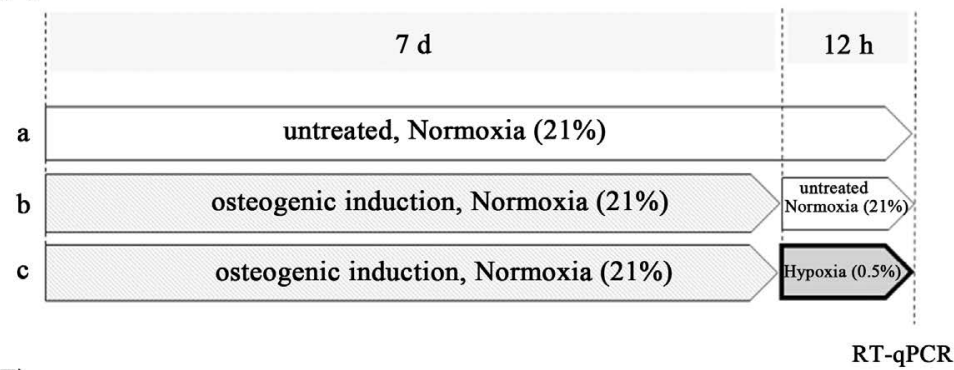

(E)

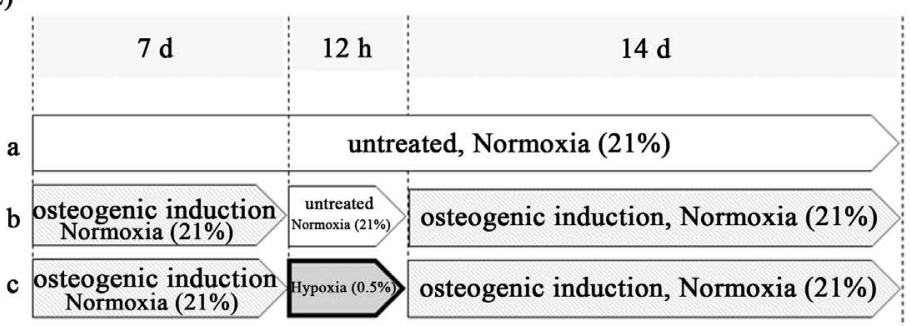

Alizarin Red S Staining

Figure 1. Different protocols tested in this study to optimize the combinational use of hypoxic exposure and osteogenic induction for enhancing the multiple osteoinductive capabilities of mesenchymal cells. (A) Protocol 1: Simultaneous application of hypoxic exposure and osteogenic induction; (B) Protocol 2: Hypoxic preconditioning followed by osteogenic induction; (C) Protocol 3: Short-time hypoxic exposure under different oxygen tensions; (D) Protocol 4: Hypoxic (post-) conditioning after osteogenic induction; (E) The cell culture protocol for Alizarin Red S staining. 


\subsubsection{Protocol 2: Effects of Hypoxic Preconditioning}

For the hypoxic preconditioning tests, cells were first cultured under hypoxia $\left(2 \% \mathrm{O}_{2}\right)$ (Figure $1(\mathrm{~B})$-c) without osteogenic induction for $72 \mathrm{~h}$ and then subjected to osteogenic induction under normoxia for $96 \mathrm{~h}$. As a control, cells were cultured under normoxia (Figure 1(B)-b) instead of hypoxia. As a baseline control, cells were cultured under normoxia (Figure 1(B)-a) for a total of $168 \mathrm{~h}$.

\subsubsection{Protocol 3: Effects of Different Low $0_{2}$ Tension Levels within $24 \mathrm{~h}$} Cells were cultured at different low $\mathrm{O}_{2}$ tensions of $0.5 \%$ (Figure $1(\mathrm{C})-\mathrm{a}-\mathrm{c}$ ), $2 \%$ (Figure 1(C)-d-f), or 5\% (Figure 1(C)-g-i), for $6 \mathrm{~h}$ (Figure 1(C)-a, Figure 1(C)-d, and Figure 1(C)-g), $12 \mathrm{~h}$ (Figure 1(C)-b, Figure 1(C)-e, and Figure 1(C)-h), and $24 \mathrm{~h}$ (Figure 1(C)-c, Figure 1(C)-f, and Figure 1(C)-i).

\subsubsection{Protocol 4: Effects of Hypoxic Post-Conditioning}

After cells were cultured with (Figure 1(D)-b and Figure 1(D)-c) or without (Figure 1(D)-a) osteogenic induction for 7 days under $21 \% \mathrm{O}_{2}$, they were subsequently cultured under either hypoxic $\left(0.5 \% \mathrm{O}_{2}\right)$ (Figure $1(\mathrm{D})$-c) or normoxic $\left(21 \% \mathrm{O}_{2}\right)$ (Figure 1(D)-a and Figure 1(D)-b) conditions for $12 \mathrm{~h}$.

\subsection{Reverse Transcription Real-Time PCR}

Total RNA was extracted from the cells using an RNeasy mini kit (Qiagen, Valencia, CA, USA), with DNase I treatment (Qiagen). RT-qPCRs were carried out using the TaqMan Fast Virus 1-Step Master Mix (Thermo Fisher Scientific) and TaqMan gene expression assays (Thermo Fisher Scientific) in the ABI PRISM 7000 sequence detection system (Thermo Fisher Scientific). The PCR cycle conditions were reverse transcription at $50^{\circ} \mathrm{C}$ for $5 \mathrm{~min}$, reverse transcription inactivation/initial denaturation at $95^{\circ} \mathrm{C}$ for $1 \mathrm{~min}$, amplification at $95^{\circ} \mathrm{C}$ for $15 \mathrm{~s}$, and 40 cycles of amplification at $60^{\circ} \mathrm{C}$ for $1 \mathrm{~min}$. In this study, expression of the following genes was measured: Runx2 (Rn01512298_m1; Thermo Fisher Scientific), Vegfa (Rn01512298_m1; Thermo Fisher Scientific), and Cxcl12 (Rn00573260_m1; Thermo Fisher Scientific). The expression level of the target gene, normalized to that of the TATA-box binding protein gene (Tbp: Rn01455648_m1; Thermo Fisher Scientific), was then calculated using the dCT method.

\subsection{Alizarin Red S Staining}

In order to examine the efficacy of Protocol 4 in enhancing the subsequent osteogenic potential of the mesenchymal cells, osteogenic induction was conducted for another 14 days under normoxia, and calcified nodule formation by the cells was evaluated by Alizarin Red S staining (Figure 1(E)). After being washed with phosphate-buffered saline, the cells were fixed at room temperature with $100 \%$ ethanol for 10 min, washed with distilled water, and then stained with 1\% Alizarin Red S solution (Wako Pure Chemical Industries) for $2 \mathrm{~min}$. After excess stain solution had been removed with distilled water, the stained area was measured 
using Image J software (Version 1.4.5I-j; National Institutes of Health, Bethesda, USA; http://rsb.info.nih.gov/ij/).

\subsection{Statistical Analysis}

Data are expressed as the mean \pm standard deviation. Statistical analyses were performed using IBM SPSS Statistics 21 (IBM Japan, Tokyo, Japan). Differences among groups were assessed by one-way analysis of variance, followed by $\mathrm{Tu}$ key's honest significant difference test. Statistics in the two groups were compared by applying Student's t-test. A p-value of less than 0.05 was considered statistically significant.

\section{Results}

\subsection{Flow Cytometric Analysis}

Cells positive for CD29 and CD90, both rat MSC markers [28], numbered 90\% or greater in the BMDCs, ATDCs, and PDLDCs (Figure 2(A) and Figure 2(B)), suggesting that the cells used in the experiments had stem cell characteristics. On the other hand, cells positive for $\mathrm{CD} 45$, a negative hematopoietic marker, were present only at $1.5 \%$ and $0.6 \%$ in the ATDCs and PDLDCs, respectively, in contrast to $27.3 \%$ in the BMDCs, implying that blood cells had remained in the BMDC culture even after subculture (Figure 2(C)).

\subsection{Expression of Runx2, Vegfa, and Cxcl12 under the Combinational Use of Hypoxic Exposure and Osteogenic Induction}

We first examined the effects of simultaneous treatment with hypoxic exposure and osteogenic induction, according to Protocol 1 (Figure 3). Compared with the normoxic control, the simultaneous treatment did not show any increase in Runx 2 expression in all three types of cells; in fact, the gene expression level under simultaneous treatment was significantly lower than that under hypoxic exposure alone in the ATDCs. The expression of Vegfa was significantly upregulated in the BMDCs and ATDCs under hypoxic exposure alone, but was remarkably downregulated by the simultaneous treatment. In contrast, in the PDLDCs, both treatments significantly increased Vegfa expression relative to that of the normoxic control. The Cxcl2 expression level was significantly dampened in the BMDCs and ATDCs under the hypoxic condition, but it remained unchanged in the PDLDCs. The results indicate that Protocol 1 has an inhibitory effect on bone regenerative activity in BMDCs and ATDCs.

Next, we tested how hypoxic preconditioning according to Protocol 2 affects gene expression (Figure 4). This second protocol did not produce any significant increase in the expression of Runx 2 and $C x c 112$ mRNAs in all three types of cells. Moreover, it significantly decreased the expression of Vegfa, indicating that hypoxic preconditioning had no effect to enhance multiple functions when subsequent normoxic culture was applied. 

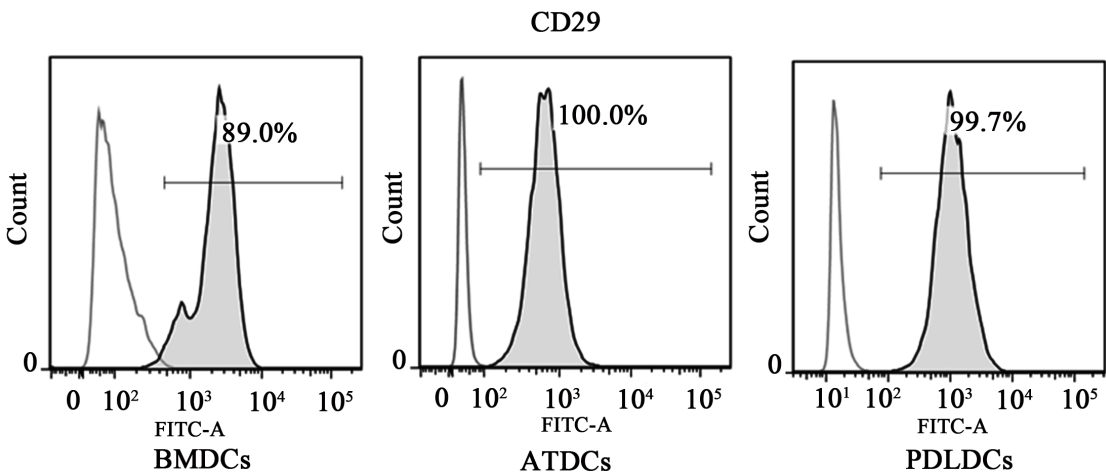

(A)
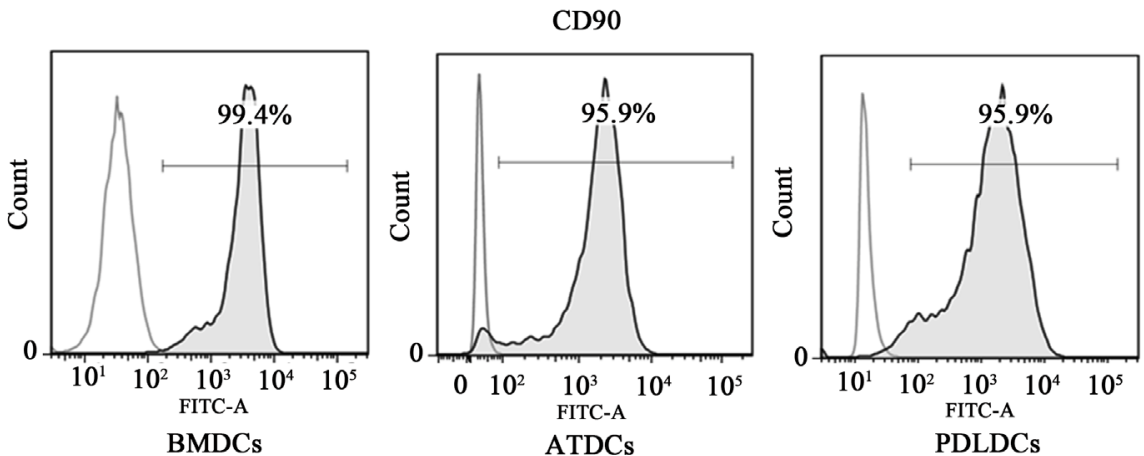

(B)

CD45
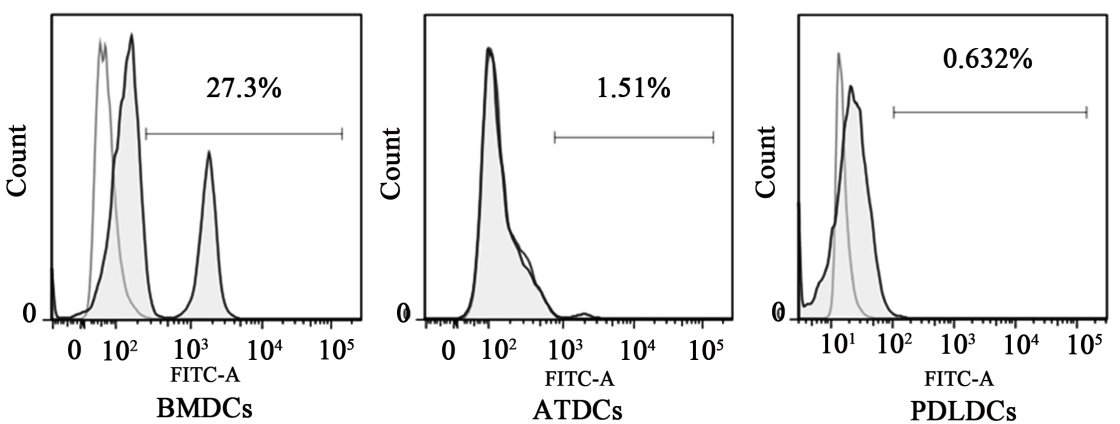

(C)

Figure 2. Flow cytometric analysis showing the immunopositive rates of CD29, CD90, and CD45 of the mesenchymal cells. Representative histograms of rat bone marrow-derived cells (BMDCs), adipose tissue-derived cells (ATDCs), and periodontal ligament-derived cells (PDLDCs).

Since the previous two protocols did not significantly enhance Runx2 expression (an osteogenic differentiation marker), we then simply assessed the effect of hypoxia by changing the $\mathrm{O}_{2}$ tension under exposures shorter than $24 \mathrm{~h}$ (Protocol 3). As expected, short hypoxic exposures did not decrease Runx2 expression under all $\mathrm{O}_{2}$ tension levels examined. In addition, Vegfa expression was significantly upregulated and reached a peak at under $0.5 \% \mathrm{O}_{2}$ for $12 \mathrm{~h}$ in the BMDCs and ATDCs. However, there were only slight effects on the expression of Cxcl12 with Protocol 3 (Figure 5). 


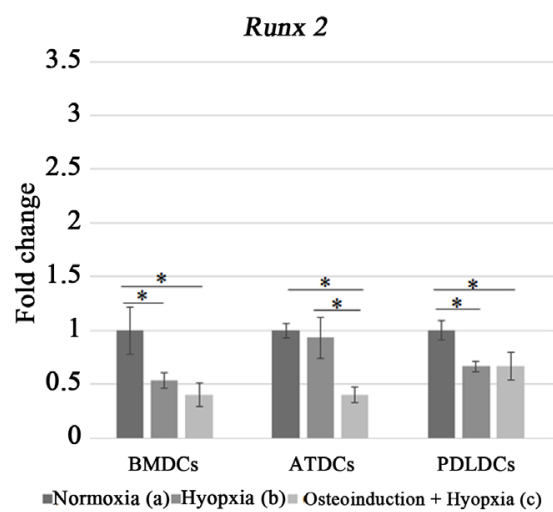

(A)

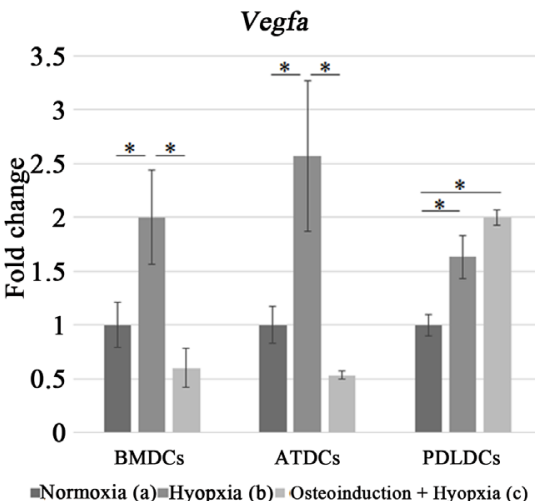

(B)

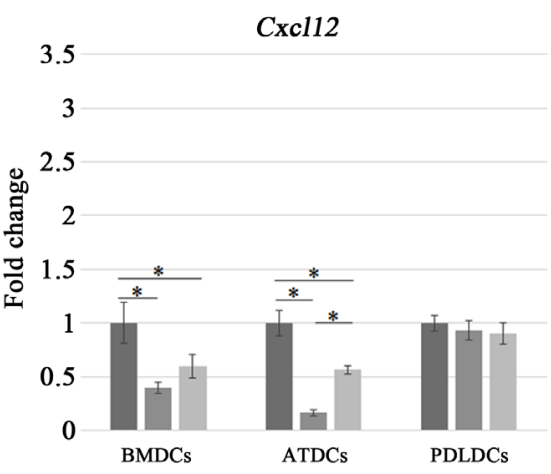

-Normoxia (a)=Hyopxia (b) $\Perp$ Osteoinduction + Hyopxia (c)

(C)

Figure 3. Average mRNA expression of Runx2 (A); Vegfa (B); and Cxcl12 (C) in rat bone marrow-derived cells (BMDCs), adipose tissue-derived cells (ATDCs), and periodontal ligament-derived cells (PDLDCs), under normoxia (A); hypoxia (2\%) (B); and simultaneous treatment of hypoxic exposure and osteogenic induction $(\mathrm{C})\left(\mathrm{n}=3{ }^{*} \mathrm{p}<0.05\right)$.

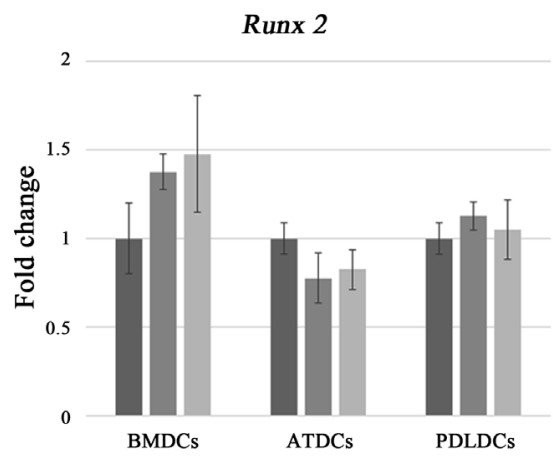

$\varpi$ Normoxia (a) $₫$ Osteoinduction (b) $₫$ Hyopxic preconditoing (c)

(A)

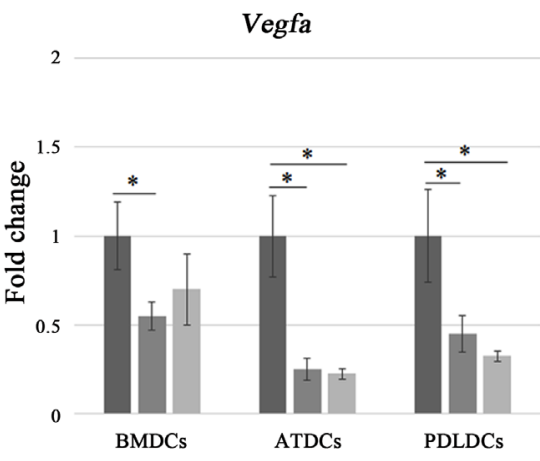

(B)

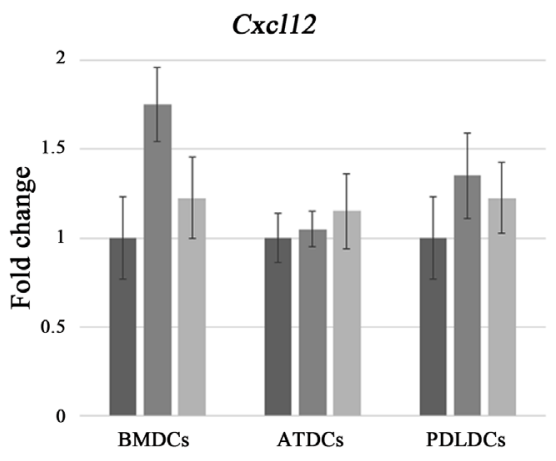

$\unrhd$ Normoxia (a) $₫$ Osteoinduction $(b)=$ Hyopxic preconditoing (c)

Figure 4. Average mRNA expression of Runx2 (A); Vegfa (B); and Cxcl12 (C) in rat bone marrow-derived cells (BMDCs), adipose tissue-derived cells (ATDCs), and periodontal ligament-derived cells (PDLDCs), under normoxia (A); osteogenic induction (B); and hypoxic preconditioning followed by osteogenic induction $(\mathrm{C})\left(\mathrm{n}=3{ }^{*}{ }^{\star} \mathrm{p}<0.05\right)$.

Finally, we examined the effect of hypoxic conditioning $\left(0.5 \% \mathrm{O}_{2}\right.$ for $\left.12 \mathrm{~h}\right)$ after osteogenic induction, according to Protocol 4. Whereas the Runx2 and Cxcl12 expression levels did not show any significant changes between osteogenic induction treatments with and without hypoxic post-conditioning in the BMDCs and ATDCs, the Runx2 expression level in the PDLDCs was significantly increased (Figure 6). In contrast, the expression level of Vegfa in all three types of cells rose drastically following hypoxic post-conditioning. Protocol 4 provided the largest fold changes of gene expression, especially of Vegfa, among the four protocols examined.

\subsection{Calcified Nodule Formation}

Using Protocol 4, the formation of calcified nodules following osteogenic induction for 2 weeks was analyzed. In the BMDCs, regardless of hypoxic post-conditioning, the area positive for Alizarin Red was larger than $90 \%$ of a 6-well 


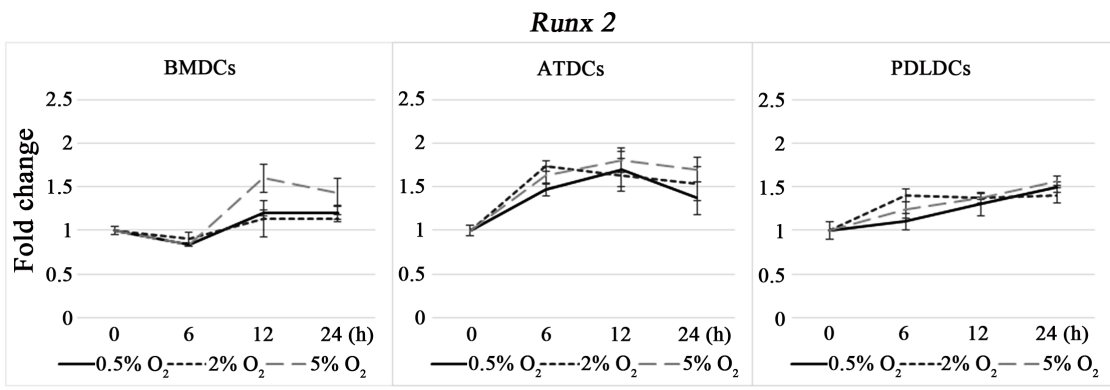

(A)

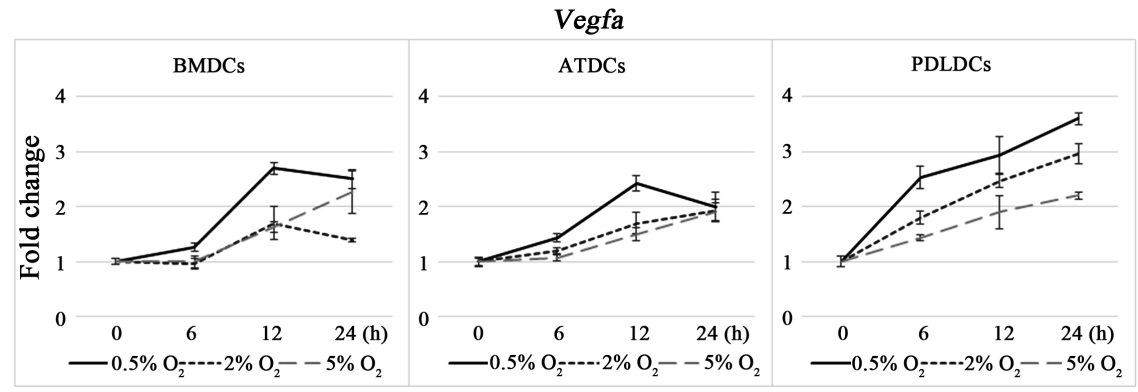

(B)

Cxc112

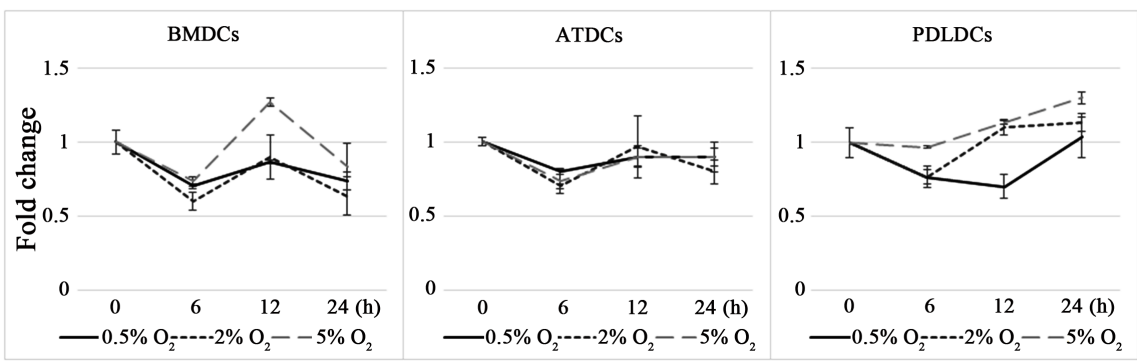

(C)

Figure 5. Average mRNA expression of Runx2 (A); Vegfa (B); and Cxcl12 (C) in bone marrow-derived cells (BMDCs), adipose tissue-derived cells (ATDCs), and periodontal ligament-derived cells (PDLDCs), under different oxygen tensions for 6, 12, and $24 \mathrm{~h} \mathrm{(n=}$ 3).

microplate well (Figure 7). In the ATDCs, however, the stained calcified area was less than $10 \%$, both with and without hypoxic post-conditioning. The PDLDCs were the only cell type revealing an affect by hypoxic post-conditioning, where the area stained was seven times larger than that without the treatment.

\section{Discussion}

A During serial cell culture, the cell population that expresses phenotypic markers for MSCs is predisposed to decline in number, resulting in the lack of effective osteogenic differentiation [29]. The present study, showing that the majority of cells obtained from the three different rat tissues expressed CD29 and CD90, confirmed that these cell populations had maintained the phenotypes of MSCs after serial cell culture. Nonetheless, our examination also detected some cells 


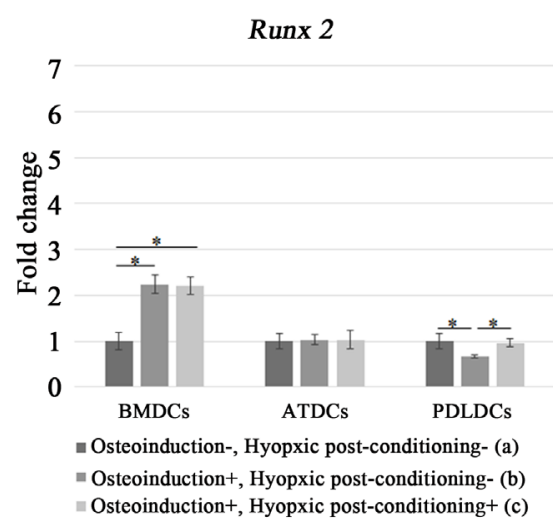

(A)

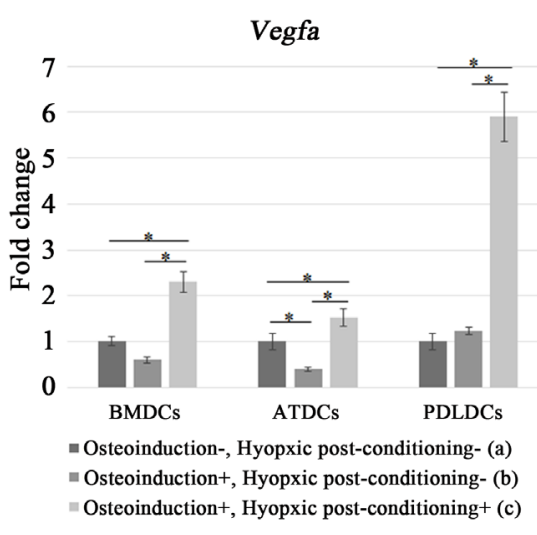

(B)

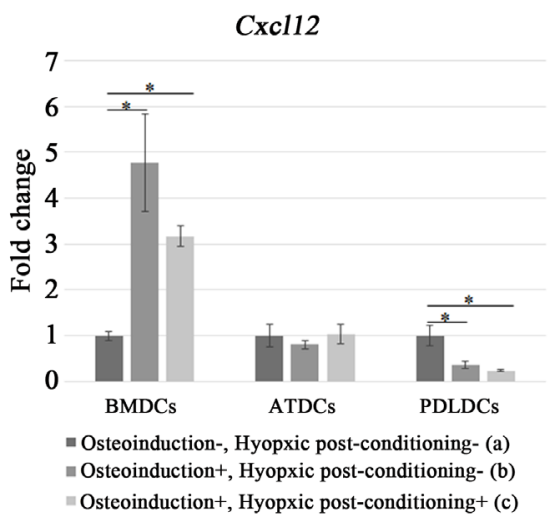

(C)

Figure 6. Average mRNA expression of Runx2(A); Vegfa (B); and Cxcl12 (C) in bone marrow-derived cells (BMDCs), adipose tissue-derived cells (ATDCs), and periodontal ligament-derived cells (PDLDCs), under normoxia (A); osteogenic induction (B); and hypoxic (post-) conditioning after osteogenic induction $(\mathrm{C})\left(\mathrm{n}=3 ;{ }^{\star} \mathrm{p}<0.05\right)$.

positive for CD45 in the BMDCs only, likely due to the presence of blood cell components after a few cell passages. In the present study, unsorted cells, instead of cells sorted by specific cell-surface markers, were used for the analyses. Thus, they were not prepared of phenotypically distinctive MSC populations at the beginning, suggesting that cell sorting might not necessarily be required prior to analyses. Although flow cytometry enables sorting of multipotent MSCs from various tissues [30] [31], it also has disadvantages in that it may cause cell damage, have a high contamination risk and cost, and reduce the number of cells retrieved [32]. Since a large number of cells can be obtained from bone marrow and adipose tissue, it is conceivable to differentiate cells without using a cell sorter in the clinical arena. Nevertheless, it is noted that contamination with cells other than MSCs may affect the efficiency of multiple osteoinductive capabilities.

In order to explore an efficient protocol for bone regeneration using a hypoxic environment, changes in cellular metabolism under hypoxia, which is a critical factor that influences responses for osteogenic differentiation, need to be considered. As an endogenous control, we used $T b p$ instead of glyceraldehyde 3-phosphate dehydrogenase ( $G a p d h)$, because previous reports have shown that the ribosomal protein L13A (Rp113A), Tbp , and beta-2 microglobulin (B2m) genes, instead of the Gapdh and actin-beta $(A c t b)$ genes, need to be chosen for hypoxic cells [33] [34]. Others also showed that $T b p$ and $R p 113 A$ are appropriate endogenous controls for studies on the induction of MSC differentiation, whereas ribosomal protein 18, $A c t b$, and B2m genes are not [35] [36]. Those are consistent with our preliminary results (data not shown). Thus, the choice of inappropriate control genes that are susceptible to hypoxia might lead to discrepancies and contradictory results among studies.

This study showed that the simultaneous application of $2 \% \mathrm{O}_{2}$ and osteogenic induction for $96 \mathrm{~h}$ did not enhance, but rather diminished, the expression levels of the three genes beneficial to rat bone regeneration, except for Vegfa expression in PDLDCs. This outcome is consistent with those of previous studies [18] 


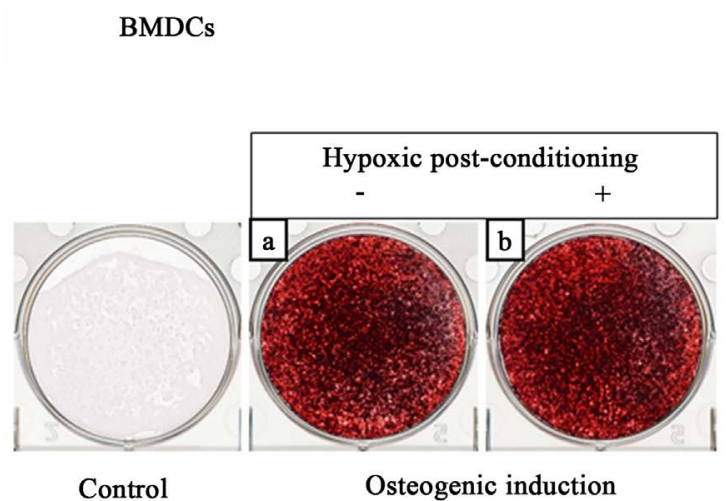

(A)

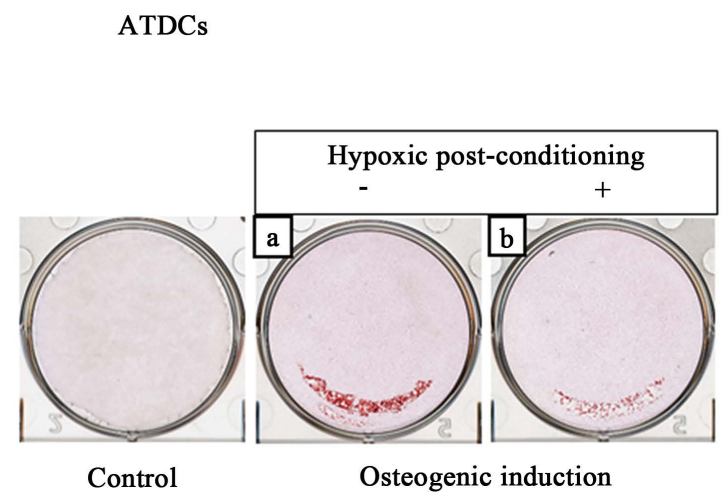

(B)

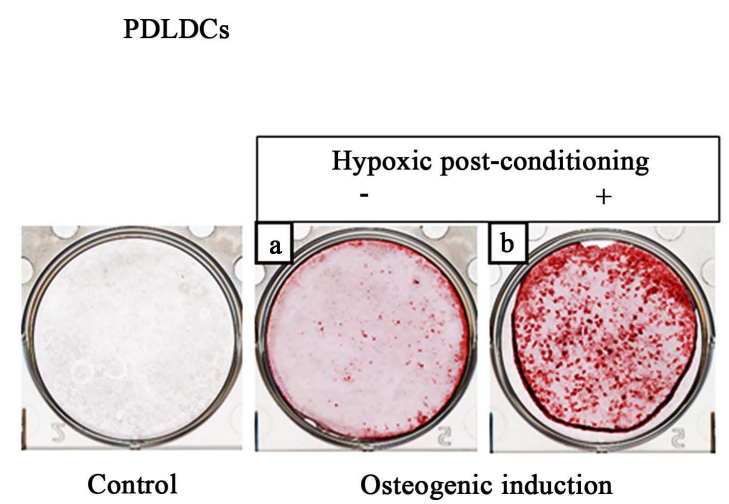

(C)
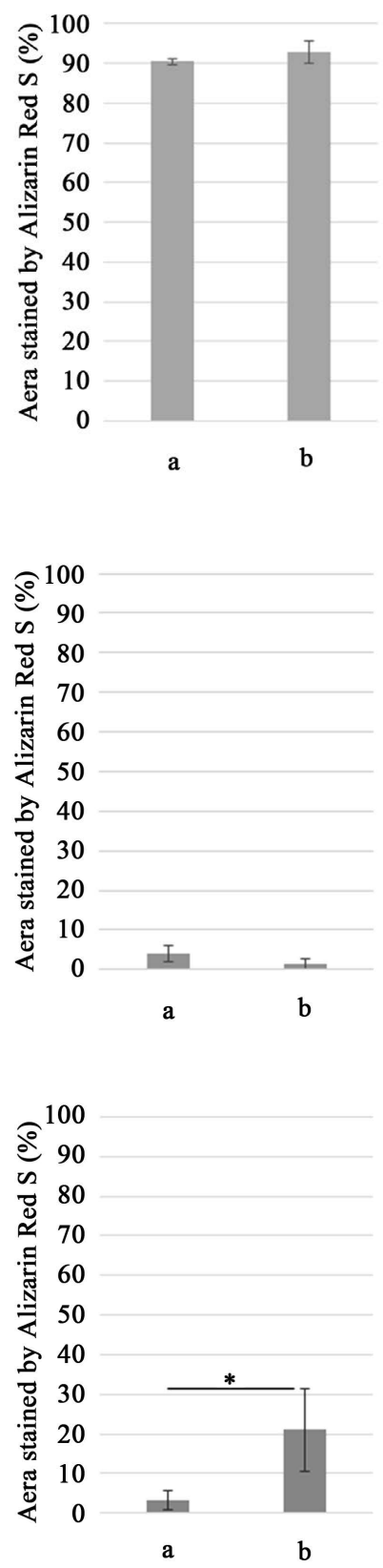

a

b

Figure 7. Formation of calcified nodules under Protocol 4 in bone marrow-derived cells (BMDCs) (A); adipose tissue-derived cells (ATDCs) (B); and periodontal ligament-derived cells (PDLDCs) (C); The left panels show a representative macroscopic view of the Alizarin Red S staining. The right panels show the percentage of area stained with Alizarin Red, measured using ImageJ software. Differences in the percentage of area stained were compared by applying Student's t-test to the results for each mesenchymal stem cell type treated without (A) and with (B) hypoxic post-conditioning $\left(\mathrm{n}=3\right.$; $\left.{ }^{\star} \mathrm{p}<0.05\right)$.

[21] [22]. However, other investigations have demonstrated that the hypoxic condition promoted osteogenic differentiation [16] [20]. This discrepancy among the different reports is likely due to the different cell culture conditions used, such as cell origin and $\mathrm{O}_{2}$ tensions, as well as exposure time and cell density seeded, 
although further clarification of this is needed. In terms of the cell culture medium composition, one study in which cells were cultured in $\alpha$-MEM containing $10 \%$ FBS (the same as our study) showed that hypoxia suppressed osteogenic differentiation [22]. However, other studies, in which cells were grown in Dulbecco's modified Eagle's medium, concluded that hypoxia promoted osteogenic differentiation [16] [20]. Thus, the type of cell culture medium may also be a factor that affects the cellular hypoxic response.

Moreover, not only did the simultaneous treatment application not significantly increase any gene expression levels, nor did the combinational use of osteogenic induction for $96 \mathrm{~h}$ with hypoxic preconditioning for $72 \mathrm{~h}$. Contrary to Protocol 1, which showed a significant increase in Vegfa expression under the hypoxic environment alone, the expression was downregulated by hypoxic preconditioning. According to Wagner et al. [37], $100 \mathrm{nM}$ of dexamethasone inhibited the nuclear transfer of hypoxia-inducible factor, resulting in the inhibition of VEGF expression. The chemical hypoxia also suppressed VEGF expression during osteogenic induction [38]. Since dexamethasone may counteract the effect of the hypoxic preconditioning that enhances Vegfa expression, this seemed to result from the subsequent osteogenic induction under normoxia, although further study to verify this is mandated.

Rupture of the vasculature within wound beds by surgical interventions causes the $\mathrm{O}_{2}$ pressure in the wound to decrease [39]. The negative impacts of Protocols 1 and 2 on osteoinductive capability could be due to the $2 \% \mathrm{O}_{2}$ tension used in those protocols, because the oxygen concentration in vivo that implies physiological "normoxia" ranges from $2 \%$ to $9 \%$ [40] [41]. As expected, the expression of Runx2 and Cxcl12 did not decrease significantly under $0.5 \% \mathrm{O}_{2}$ (severe hypoxia) within $24 \mathrm{~h}$. When we applied "hypoxic conditioning" after osteogenic induction under Protocol 4, the expression levels of the three different genes were restored compared with the levels under Protocol 2. In addition, the fold changes of expression level of the three genes were larger in Protocol 4 than in Protocol 1, although the expression patterns did not change significantly. Furthermore, under Protocol 4, the formation of calcified nodules was distinctly different among the three types of cells, suggesting there are differential responses to hypoxia and osteogenic induction, with varying levels, among MSCs of different tissue origins. This is consistent with a previous investigation that demonstrated the enhanced osteogenic capacity of PDLDCs by the paracrine effect of VEGF-A [42].

This study clearly showed that hypoxic responses vary among cells that originated from different mesenchymal tissues. For instance, the intrinsic gene expression levels of Vegfa and Cxcl12 are higher in BMDCs than in ATDCs [43] [44]. Since the hypoxic responses of MSCs are likely to be tissue specific, the origin of the MSCs needs to be considered before their use as therapeutic agents.

\section{Conclusion}

In summary, the largest fold changes of expression level of the three genes per- 
taining to multiple osteoinductive capabilities were induced by Protocol 4 ( $0.5 \%$ $\mathrm{O}_{2}$ hypoxic post-conditioning with osteogenic induction), although the Vegfa and $C x c 112$ expression levels were the most and least susceptible to this protocol, respectively. Since there were differential responses of the mineralized nodule formation among mesenchymal cells from different tissues, the optimal protocol developed should be specific to the origin of the mesenchymal cells. Although further in vivo studies on protein expression and cell behaviors are necessary, manipulating mesenchymal cells with hypoxic exposure after osteogenic induction before transplantation could have potential for improving their therapeutic efficiency in bone regeneration.

\section{Ethical Approval}

This experiment was approved by the Niigata University Animal Experimentation Ethics Committee (Approval No. 71-4), and performed according to the animal experiment rules of Niigata University.

\section{Conflict of Interest}

There is no conflict of interest.

\section{Acknowledgements}

The Authors would like to thank all the staff in the divisions of Oral Anatomy, Bio-prosthodontics, Biomimetics, and Oral Reconstructive Surgery at Niigata University Graduate School of Medical and Dental Sciences. This work was supported by a Grant-in-Aid for JSPS Fellows (15J01649).

\section{References}

[1] Chiapasco, M., Colletti, G., Romeo, E., Zaniboni, M. and Brusati, R. (2008) Long-Term Results of Mandibular Reconstruction with Autogenous Bone Grafts and Oral Implants after Tumor Resection. Clinical Oral Implants Research, 19, 1074-1080. https://doi.org/10.1111/j.1600-0501.2008.01542.x

[2] Goh, B.T., Lee, S., Tideman, H. and Stoelinga, P.J. (2008) Mandibular Reconstruction in Adults: A Review. International Journal of Oral \& Maxillofacial Surgery, 37, 597-605. https://doi.org/10.1016/j.ijom.2008.03.002

[3] Handschel, J., Hassanyar, H., Depprich, R.A., Ommerborn, M.A., Sproll, K.C., Hofer, M., et al. (2011) Nonvascularized Iliac Bone Grafts for Mandibular Reconstruction-Requirements and Limitations. In Vivo, 25, 795-799.

[4] Herford, A.S., Stoffella, E. and Tandon, R. (2011) Reconstruction of Mandibular Defects Using Bone Morphogenic Protein: Can Growth Factors Replace the Need for Autologous Bone Grafts? A Systematic Review of the Literature. Plastic Surgery International, 2011, Article ID: 165824. https://doi.org/10.1155/2011/165824

[5] Chanchareonsook, N., Junker, R., Jongpaiboonkit, L. and Jansen, J.A. (2014) Tissue-Engineered Mandibular Bone Reconstruction for Continuity Defects: A Systematic Approach to the Literature. Tissue Engineering Part B: Reviews, 20, 147-162. https://doi.org/10.1089/ten.teb.2013.0131

[6] Khojasteh, A., Behnia, H., Naghdi, N., Esmaeelinejad, M., Alikhassy, Z. and Stevens, 
M. (2013) Effects of Different Growth Factors and Carriers on Bone Regeneration: A Systematic Review. Oral Surgery, Oral Medicine, Oral Pathology, Oral Radiology, 116, e405-e423. https://doi.org/10.1016/j.oooo.2012.01.044

[7] Torroni, A. (2009) Engineered Bone Grafts and Bone Flaps for Maxillofacial Defects: State of the Art. Journal of Oral and Maxillofacial Surgery, 67, 1121-1127. https://doi.org/10.1016/j.joms.2008.11.020

[8] Miura, M., Miura, Y., Sonoyama, W., Yamaza, T., Gronthos, S. and Shi, S. (2006) Bone Marrow-Derived Mesenchymal Stem Cells for Regenerative Medicine in Craniofacial Region. Oral Diseases, 12, 514-522. https://doi.org/10.1111/j.1601-0825.2006.01300.x

[9] Kaku, M., Akiba, Y., Akiyama, K., Akita, D. and Nishimura, M. (2015) Cell-Based Bone Regeneration for Alveolar Ridge Augmentation-Cell Source, Endogenous Cell Recruitment and Immunomodulatory Function. Journal of Prosthodontic Research, 59, 96-112. https://doi.org/10.1016/j.jpor.2015.02.001

[10] Yu, S.P., Wei, Z. and Wei, L. (2013) Preconditioning Strategy in Stem Cell Transplantation Therapy. Translational Stroke Research, 4, 76-88. https://doi.org/10.1007/s12975-012-0251-0

[11] Sart, S., Ma, T. and Li, Y. (2014) Preconditioning Stem Cells for In Vivo Delivery. BioResearch Open Access, 3, 137-149. https://doi.org/10.1089/biores.2014.0012

[12] Lu, Z., Wang, G., Dunstan, C.R., Chen, Y., Lu, W.Y., Davies, B., et al. (2013) Activation and Promotion of Adipose Stem Cells by Tumour Necrosis Factor- $\alpha$ Preconditioning for Bone Regeneration. Journal of Cellular Physiology, 228, 1737-1744. https://doi.org/10.1002/jcp.24330

[13] Tavassol, F., Kampmann, A., Lindhorst, D., Schumann, P., Kokemüller, H., Bormann, K.H., et al. (2011) Prolongated Survival of Osteoblast-Like Cells on Biodegradable Scaffolds by Heat Shock Preconditioning. Tissue Engineering Part A, 17, 1935-1943. https://doi.org/10.1089/ten.tea.2010.0603

[14] Kubo, M., Li, T.S., Kurazumi, H., Takemoto, Y., Ohshima, M., Murata, T., et al. (2012) Hypoxic Preconditioning Enhances Angiogenic Potential of Bone Marrow Cells with Aging-Related Functional Impairment. Circulation Journal, 76, 986-994. https://doi.org/10.1253/circj.CJ-11-0605

[15] Wei, L., Fraser, J.L., Lu, Z.Y., Hu, X. and Yu, S.P. (2012) Transplantation of Hypoxia Preconditioned Bone Marrow Mesenchymal Stem Cells Enhances Angiogenesis and Neurogenesis after Cerebral Ischemia in Rats. Neurobiology of Disease, 46, 635-645. https://doi.org/10.1016/j.nbd.2012.03.002

[16] Wagegg, M., Gaber, T., Lohanatha, F.L., Hahne, M., Strehl, C., Fangradt, M., et al. (2012) Hypoxia Promotes Osteogenesis but Suppresses Adipogenesis of Human Mesenchymal Stromal Cells in a Hypoxia-Inducible Factor-1 Dependent Manner. PLoS ONE, 7, e46483. https://doi.org/10.1371/journal.pone.0046483

[17] Potier, E., Ferreira, E., Andriamanalijaona, R., Pujol, J.P., Oudina, K., Logeart-Avramoglou, D., et al. (2007) Hypoxia Affects Mesenchymal Stromal Cell Osteogenic Differentiation and Angiogenic Factor Expression. Bone, 40, 1078-1087.

https://doi.org/10.1016/j.bone.2006.11.024

[18] Chung, D.J., Hayashi, K., Toupadakis, C.A., Wong, A. and Yellowley, C.E. (2012) Osteogenic Proliferation and Differentiation of Canine Bone Marrow and Adipose Tissue Derived Mesenchymal Stromal Cells and the Influence of Hypoxia. Research in Veterinary Science, 92, 66-75. https://doi.org/10.1016/j.rvsc.2010.10.012

[19] Hung, S.P., Ho, J.H., Shih, Y.R., Lo, T. and Lee, O.K. (2012) Hypoxia Promotes Proliferation and Osteogenic Differentiation Potentials of Human Mesenchymal Stem 
Cells. Journal of Orthopaedic Research, 30, 260-266.

https://doi.org/10.1002/jor.21517

[20] Lennon, D.P., Edmison, J.M. and Caplan, A.I. (2001) Cultivation of Rat Marrow-Derived Mesenchymal Stem Cells in Reduced Oxygen Tension: Effects on in Vitro and in Vivo Osteochondrogenesis. Journal of Cellular Physiology, 187, 345-355. https://doi.org/10.1002/jcp.1081

[21] Volkmer, E., Kallukalam, B.C., Maertz, J., Otto, S., Drosse, I., Polzer, H., et al. (2010) Hypoxic Preconditioning of Human Mesenchymal Stem Cells Overcomes Hypoxia-Induced Inhibition of Osteogenic Differentiation. Tissue Engineering Part $A, 16,153-164$. https://doi.org/10.1089/ten.tea.2009.0021

[22] Wang, Y., Li, J., Wang, Y., Lei, L., Jiang, C., An, S., et al. (2012) Effects of Hypoxia on Osteogenic Differentiation of Rat Bone Marrow Mesenchymal Stem Cells. Molecular and Cellular Biochemistry, 362, 25-33.

https://doi.org/10.1007/s11010-011-1124-7

[23] Wen, Y., Jiang, B., Cui, J., Li, G., Yu, M., Wang, F., et al. (2013) Superior Osteogenic Capacity of Different Mesenchymal Stem Cells for Bone Tissue Engineering. Oral Surgery, Oral Medicine, Oral Pathology, Oral Radiology, 116, e324-e332. https://doi.org/10.1016/j.oooo.2012.02.024

[24] Liao, H.T. and Chen, C.T. (2014) Osteogenic Potential: Comparison between Bone Marrow and Adipose-Derived Mesenchymal Stem Cells. World Journal of Stem Cells, 6, 288-295. https://doi.org/10.4252/wjsc.v6.i3.288

[25] Morcos, M.W., Al-Jallad, H. and Hamdy, R. (2015) Comprehensive Review of Adipose Stem Cells and Their Implication in Distraction Osteogenesis and Bone Regeneration. BioMed Research International, 2015, Article ID: 842975. https://doi.org/10.1155/2015/842975

[26] Yu, B.-H., Zhou, Q. and Wang, Z.-L. (2014) Comparison of Tissue-Engineered Bone from Different Stem Cell Sources for Maxillary Sinus Floor Augmentation: A Study in a Canine Model. Journal of Oral and Maxillofacial Surgery, 72, 1084-1092. https://doi.org/10.1016/j.joms.2013.12.024

[27] Egusa, H., Sonoyama, W., Nishimura, M., Atsuta, I. and Akiyama, K. (2012) Stem Cells in Dentistry-Part I: Stem Cell Sources. Journal of Prosthodontic Research, 56, 151-165. https://doi.org/10.1016/j.jpor.2012.06.001

[28] Fafián-Labora, J., Fernández-Pernas, P., Fuentes, I., De Toro, J., Oreiro, N., Sangiao-Alvarellos, S., et al. (2015) Influence of Age on Rat Bone-Marrow Mesenchymal Stem Cells Potential. Scientific Reports, 5, Article No. 16765.

[29] Lee, J.S., Park, J.C., Kim, T.W., Jung, B.J., Lee, Y., Shim, E.K., et al. (2015) Human Bone Marrow Stem Cells Cultured under Hypoxic Conditions Present Altered Characteristics and Enhanced in Vivo Tissue Regeneration. Bone, 78, 34-45.

https://doi.org/10.1016/j.bone.2015.04.044

[30] Mabuchi, Y., Morikawa, S., Harada, S., Niibe, K., Suzuki, S., Renault-Mihara, F., et al. (2015) LNGFR $^{+}$THY $-1^{+}$VCAM- ${ }^{\text {hi+ }}$ Cells Reveal Functionally Distinct Subpopulations in Mesenchymal Stem Cells. Stem Cell Reports, 1, 152-165. https://doi.org/10.1016/j.stemcr.2013.06.001

[31] Nery, A.A., Nascimento, I.C., Glaser, T., Bassaneze, V., Krieger, J.E. and Ulrich, H. (2013) Human Mesenchymal Stem Cells: From Immunophenotyping by Flow Cytometry to Clinical Applications. Cytometry Part A, 83A, 48-61. https://doi.org/10.1002/cyto.a.22205

[32] Autebert, J., Coudert, B., Bidard, F.C., Pierga, J.Y., Descroix, S., Malaquin, L., et al. (2012) Microfluidic: An Innovative Tool for Efficient Cell Sorting. Methods, 57, 
297-307. https://doi.org/10.1016/j.ymeth.2012.07.002

[33] Baddela, V.S., Baufeld, A., Yenuganti, V.R., Vanselow, J. and Singh, D. (2014) Suitable Housekeeping Genes for Normalization of Transcript Abundance Analysis by Real-Time RT-PCR in Cultured Bovine Granulosa Cells during Hypoxia and Differential Cell Plating Density. Reproductive Biology and Endocrinology, 12, 118. https://doi.org/10.1186/1477-7827-12-118

[34] Foldager, C.B., Munir, S., Ulrik-Vinther, M., Søballe, K., Bünger, C. and Lind, M. (2009) Validation of Suitable House Keeping Genes for Hypoxia-Cultured Human Chondrocytes. BMC Molecular Biology, 10, 94. https://doi.org/10.1186/1471-2199-10-94

[35] Quiroz, F.G., Posada, O.M., Gallego-Perez, D., Higuita-Castro, N., Sarassa, C., Hansford, D.J., et al. (2010) Housekeeping Gene Stability Influences the Quantification of Osteogenic Markers during Stem Cell Differentiation to the Osteogenic Lineage. Cytotechnology, 62, 109-120. https://doi.org/10.1007/s10616-010-9265-1

[36] Ragni, E., Viganò, M., Rebulla, P., Giordano, R. and Lazzari, L. (2013) What Is beyond a qRT-PCR Study on Mesenchymal Stem Cell Differentiation Properties: How to Choose the Most Reliable Housekeeping Genes. Journal of Cellular and Molecular Medicine, 17, 168-180. https://doi.org/10.1111/j.1582-4934.2012.01660.x

[37] Wagner, A.E., Huck, G., Stiehl, D.P., Jelkmann, W. and Hellwig-Bürgel, T. (2008) Dexamethasone Impairs Hypoxia-Inducible Factor-1 Function. Biochemical and Biophysical Research Communications, 372, 336-340. https://doi.org/10.1016/j.bbrc.2008.05.061

[38] Wu, Y., Lucia, K., Lange, M., Kuhlen, D., Stalla, G.K. and Renner, U. (2014) Hypoxia Inducible Factor-1 Is Involved in Growth Factor, Glucocorticoid and Hypoxia Mediated Regulation of Vascular Endothelial Growth Factor-A in Human Meningiomas. Journal of Neuro-Oncology, 119, 263-273. https://doi.org/10.1007/s11060-014-1503-5

[39] Kang, S., Lee, D., Theusch, B.E., Arpey, C.J. and Brennan, T.J. (2013) Wound Hypoxia in Deep Tissue after Incision in Rats. Wound Repair and Regeneration, 21, 730-739. https://doi.org/10.1111/wrr.12081

[40] Mohyeldin, A., Garzón-Muvdi, T. and Quiñones-Hinojosa, A. (2012) Oxygen in Stem Cell Biology: A Critical Component of the Stem Cell Niche. Cell Stem Cell, 7, 150-161. https://doi.org/10.1016/j.stem.2010.07.007

[41] Sen, C.K., Khanna, S. and Roy, S. (2006) Perceived Hyperoxia: Oxygen-Induced Remodeling of the Reoxygenated Heart. Cardiovascular Research, 71, 280-288. https://doi.org/10.1016/j.cardiores.2006.01.003

[42] Wu, Y., Cao, H., Yang, Y., Zhou, Y., Gu, Y., Zhao, X., et al. (2013) Effects of Vascular Endothelial Cells on Osteogenic Differentiation of Noncontact Co-Cultured Periodontal Ligament Stem Cells under Hypoxia. Journal of Periodontal Research, 48, 52-65. https://doi.org/10.1111/j.1600-0765.2012.01503.x

[43] Dmitrieva, R.I., Minullina, I.R., Bilibina, A.A., Tarasova, O.V., Anisimov, S.V. and Zaritskey, A.Y. (2012) Bone Marrow- and Subcutaneous Adipose Tissue-Derived Mesenchymal Stem Cells: Differences and Similarities. Cell Cycle, 11, 377-383. https://doi.org/10.4161/cc.11.2.18858

[44] Nakanishi, C., Nagaya, N., Ohnishi, S., Yamahara, K., Takabatake, S., Konno, T., et al. (2011) Gene and Protein Expression Analysis of Mesenchymal Stem Cells Derived from Rat Adipose Tissue and Bone Marrow. Circulation Journal, 75, 2260-2268. 